\title{
Unconventional superconductivity in the layered iron germanide $\mathrm{YFe}_{2} \mathrm{Ge}_{2}$
}

\author{
Jiasheng Chen, ${ }^{1}$ Konstantin Semeniuk, ${ }^{1}$ Zhuo Feng, ${ }^{2}$ Pascal Reiss,${ }^{1}$ Philipp Brown,${ }^{1}$ \\ Yang Zou, ${ }^{1}$ Peter W. Logg, ${ }^{1}$ Giulio I. Lampronti, ${ }^{3}$ and F. Malte Grosche ${ }^{1, *}$ \\ ${ }^{1}$ Cavendish Laboratory, University of Cambridge, Cambridge CB3 OHE, United Kingdom \\ ${ }^{2}$ London Centre of Nanotechnology, University College London, London WC1H OAH, United Kingdom \\ ${ }^{3}$ Department of Earth Sciences, University of Cambridge, Cambridge CB2 3EQ, United Kingdom
}

(Dated: February 22, 2016)

The iron-based intermetallic $\mathrm{YFe}_{2} \mathrm{Ge}_{2}$ stands out among transition metal compounds for its high Sommerfeld coefficient of the order of $100 \mathrm{~mJ} /\left(\mathrm{molK}^{2}\right)$, which signals strong electronic correlations. A new generation of high quality samples of $\mathrm{YFe}_{2} \mathrm{Ge}_{2}$ show superconducting transition anomalies below $1.8 \mathrm{~K}$ in thermodynamic, magnetic and transport measurements, establishing that superconductivity is intrinsic in this layered iron compound outside the known superconducting iron pnictide or chalcogenide families. The Fermi surface geometry of $\mathrm{YFe}_{2} \mathrm{Ge}_{2}$ resembles that of $\mathrm{KFe}_{2} \mathrm{As}_{2}$ in the high pressure collapsed tetragonal phase, in which superconductivity at temperatures as high as $10 \mathrm{~K}$ has recently been reported, suggesting an underlying connection between the two systems.

Since the discovery of superconductivity in LaFePO [1], numerous iron-based superconductors have been identified within diverse structure families, all of which combine iron with a group-V (pnictogen) or group-VI (chalcogen) element. Unconventional superconductivity is extremely rare among transition metal compounds outside these layered iron systems and the cuprates, and it is almost universally associated with highly anisotropic electronic properties and nearly 2D Fermi surface geometries [2-4]. This contrasts with the comparatively isotropic, 3D electronic structure of the iron germanide $\mathrm{YFe}_{2} \mathrm{Ge}_{2}$ [5-7], in which resistive and magnetic signatures of superconductivity have recently been reported $[8,9] . \quad \mathrm{YFe}_{2} \mathrm{Ge}_{2}$ shares key properties with the alkali metal iron arsenides $(\mathrm{K} / \mathrm{Rb} / \mathrm{Cs}) \mathrm{Fe}_{2} \mathrm{As}_{2}$ [10-14]: it has the same $\mathrm{ThCr}_{2} \mathrm{Si}_{2}$ structure, featuring square lattice iron layers, its low temperature heat capacity Sommerfeld coefficient is similarly enhanced, and antiferromagnetic order can be induced by chemical substitution [15]. Recent x-ray absorption and photoemission studies have demonstrated the presence of large fluctuating Fe-moments in $\mathrm{YFe}_{2} \mathrm{Ge}_{2}$ [16], suggesting that this system is close to the border of magnetism. There is an important difference, however: although $\mathrm{YFe}_{2} \mathrm{Ge}_{2}$ appears at first sight to be isoelectronic to the alkali metal iron arsenide superconductors, the existence of Ge-Ge bonds in $\mathrm{YFe}_{2} \mathrm{Ge}_{2}$, contrasting with the absence of As-As bonds in the arsenides, causes the Fe oxidation state to differ from that of the arsenides [7].

Because initial experimental studies have failed to produce thermodynamic evidence for a bulk superconducting transition in $\mathrm{YFe}_{2} \mathrm{Ge}_{2}$, the possibility of filamentary superconductivity from alien phases, advanced also in [17], has held back further work on this material. Here, we present transport, magnetic and thermodynamic evidence for a bulk superconducting transition in $\mathrm{YFe}_{2} \mathrm{Ge}_{2}$ obtained in a new generation of high quality samples. This confirms the intrinsic nature of superconductivity in $\mathrm{YFe}_{2} \mathrm{Ge}_{2}$ and motivates further investigations into the nature of its unconventional superconducting and anoma-

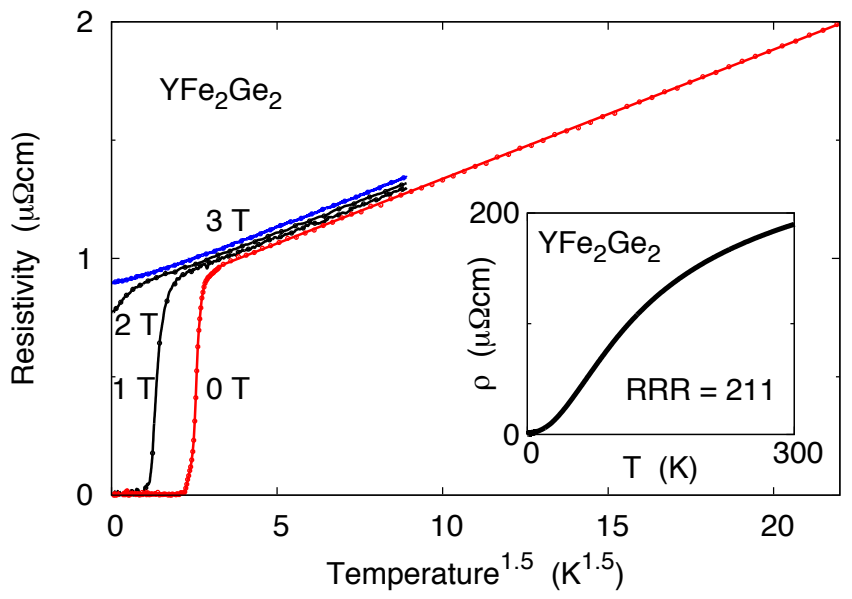

FIG. 1. Electrical resistivity of $\mathrm{YFe}_{2} \mathrm{Ge}_{2}$ versus temperature, displaying a sharp superconducting drop of the resistivity with mid-point at $1.83 \mathrm{~K}$ below a $T^{3 / 2}$ normal state temperature dependence. (inset) Temperature dependence of the resistivity up to room temperature.

lous normal state. We note, also, the striking similarity between the electronic structure of $\mathrm{YFe}_{2} \mathrm{Ge}_{2}$ and that of $\mathrm{KFe}_{2} \mathrm{As}_{2}$ in the pressure induced collapsed tetragonal state, which suggests that the two systems share a common pairing mechanism.

Polycrystalline ingots of $\mathrm{YFe}_{2} \mathrm{Ge}_{2}$ were obtained by radio frequency induction melting on a water-cooled copper boat in an argon atmosphere. To circumvent the formation of stable $\mathrm{Y}-\mathrm{Ge}$ alloys, $\mathrm{YFe}_{2}$ was first grown from the elements (Y 3N, Fe 4N). Together with elemental Ge (6N) and small amounts of extra $\mathrm{Fe}$ or $\mathrm{Y}$ this was then used to grow stoichiometric as well as slightly off-stoichiometric $\mathrm{YFe}_{2} \mathrm{Ge}_{2}$. The melt was quenched and then annealed in argon at $1250^{\circ} \mathrm{C}$ for 1 hour, followed by further annealing in vacuum at $800^{\circ} \mathrm{C}$ for 8 days. More than 20 ingots with varying nominal starting compositions have been produced, reaching up to four times higher residual resistance ratios (RRR) than those reported previously [8]. 
X-ray studies [18] show that our samples are $97.5-99.5 \%$ phase pure, the dominant impurity phase being a ferromagnetic Fe-Ge alloy with composition approximately $\mathrm{Fe}_{0.85} \mathrm{Ge}_{0.15}$ and Curie temperature $\sim 700{ }^{\circ} \mathrm{C}[22]$. The highest quality samples, including those shown in Figs. 1 and 3, were grown from an iron-rich melt with nominal composition $\mathrm{YFe}_{2.1} \mathrm{Ge}_{2}$, and analysis of impurity phase content [18] indicates that their actual composition is $\mathrm{YFe}_{2+x} \mathrm{Ge}_{2}$ with $0<x<0.03$. The electrical resistance was measured using a standard four-terminal AC technique in an adiabatic demagnetisation refrigerator to $0.1 \mathrm{~K}$ and in a Quantum Design Physical Properties Measurement System (PPMS) to $<0.4 \mathrm{~K}$. Data were scaled at $300 \mathrm{~K}$ to the published high temperature resistivity [5]. The specific heat capacity was measured in a PPMS to below $0.4 \mathrm{~K}$. The magnetisation data was acquired in a Cryogenic SQUID magnetometer to below $0.31 \mathrm{~K}$ and was corrected for the effect of demagnetising fields by approximating the sample shape as ellipsoidal. The electronic structure was calculated using the Generalized Gradient Approximation [23] with Wien2k [24]. Experimentally determined lattice parameters were used for $\mathrm{YFe}_{2} \mathrm{Ge}_{2}$ and for $\mathrm{KFe}_{2} \mathrm{As}_{2}$ at ambient pressure and at a pressure of $21 \mathrm{GPa}$ [25] (Tab. I). $R k_{\max }=7.5$ and 100,000 $k$-points were used (6768 $k$-points in the irreducible Brillouin zone), and spin orbit coupling and relativistic local orbitals were included. The fractional internal position of the Ge or As layer, $z$, the only free internal coordinate, was optimised numerically, resulting in $z=0.3699$ in $\mathrm{YFe}_{2} \mathrm{Ge}_{2}$ and $z=0.3675$ in collapsed tetragonal $\mathrm{KFe}_{2} \mathrm{As}_{2}$.

At temperatures below $10 \mathrm{~K}$, the electrical resistivity of all samples of $\mathrm{YFe}_{2} \mathrm{Ge}_{2}$ displays an unconventional power-law temperature dependence of the form $\rho(T) \simeq \rho_{0}+A T^{3 / 2}$ (Fig. 1). This suggests Fermi liquid breakdown similar to that observed in other transition metal compounds such as $\mathrm{MnSi}, \mathrm{ZrZn}_{2}$ and $\mathrm{NbFe}_{2}$ near the threshold of magnetic order [26-29] and is reminiscent of the $T^{3 / 2}$ power-law temperature dependences reported in early studies of $\mathrm{KFe}_{2} \mathrm{As}_{2}$ [30] and $\mathrm{CsFe}_{2} \mathrm{As}_{2}$ [31]. The dependence on residual resistivity of the resistivity exponent, which is reported to reach the Fermi liquid value of 2 in the cleanest samples of $\mathrm{KFe}_{2} \mathrm{As}_{2}$ [32], might be attributed to the hot spot/cold spot scenario for scattering from nearly critical antiferromagnetic fluctuations $[33,34]$.

Although most samples show resistive superconducting transitions (Fig. 1), the midpoint transition temperature $T_{c}$ and the transition width depend strongly on growth conditions. The highest transition temperatures and narrowest transitions were observed in those samples which also have the highest RRR (Fig. 2). Full resistive transitions are observed in most samples with RRR values exceeding 20 , and the value of $T_{c}$, which hovers around $1.3 \mathrm{~K}$ for RRR values of up to 70 (blue shaded region in Fig. 2), steps up to around $1.8 \mathrm{~K}$ for RRR $>70$ (red shaded region in Fig. 2). This analysis of our data does

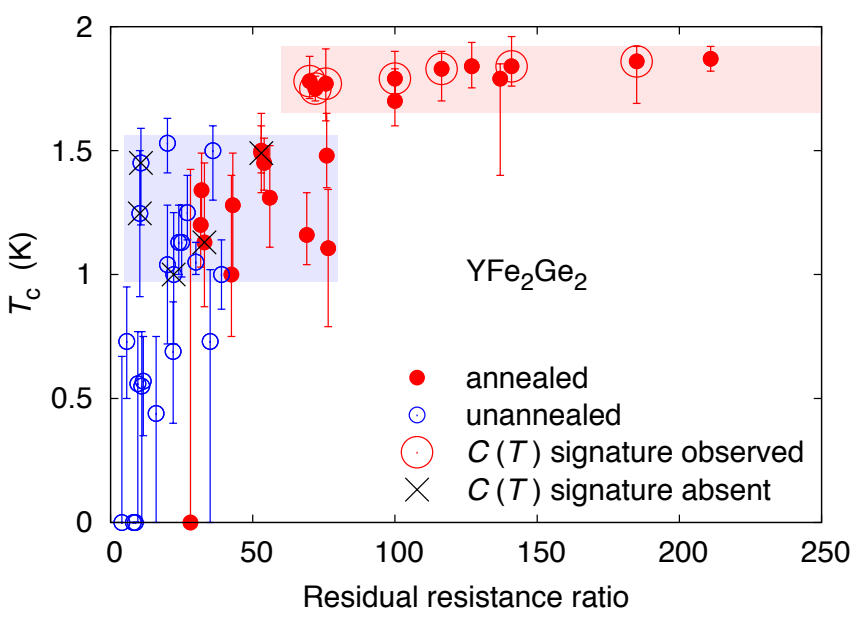

FIG. 2. Correlation between superconducting transition temperature $T_{c}$ and residual resistance ratio $\mathrm{RRR}=$ $\rho(300 \mathrm{~K}) / \rho(2 \mathrm{~K})$. Data points and error bars show resistive transition mid-point temperature and transition width, determined by an $80 \% / 20 \%$ criterion. Samples annealed at $800^{\circ} \mathrm{C}$ (filled red circles) can reach higher RRR and higher $T_{c}$ than samples which have not been annealed at $800^{\circ} \mathrm{C}$ (unfilled blue circles). The $T$-dependent heat capacity $C(T)$ was measured for a subset of the samples. Large red circles mark those cases, in which a clear $C(T)$ anomaly was observed, whereas the absence of such an anomaly is marked with black crosses. The $C(T)$ anomalies occur at about $1 \mathrm{~K}$, as is illustrated in Fig. 3 for the sample with $\mathrm{RRR}=185$. Shaded areas illustrate the step in $T_{c}$ discussed in the text.

not yet take into account other underlying correlations which may affect RRR and $T_{c}$, such as slight variations in composition or the effect of annealing on microscopic inhomogeneity. It does, however, suggest that the samples most likely to display bulk superconductivity may be found towards the high RRR end of Fig. 2 .

Neither flux-grown samples of $\mathrm{YFe}_{2} \mathrm{Ge}_{2}$ with $\mathrm{RRR}<$ 60 nor our previous generation of induction furnacegrown samples have shown a superconducting anomaly in the $T$ - dependence of the heat capacity, $C(T)[8,9]$. By contrast, our new generation of samples display clear $C(T)$ anomalies below the resistive transition temperature $T_{c}$. Testing a number of samples with different RRR reveals superconducting $C(T)$ anomalies in every tested sample for which the resistive $T_{c}$ falls into the higher, red shaded region mentioned above (large circles in Fig. 2), whereas every other tested sample shows no superconducting $C(T)$ anomaly (crosses in Fig. 2).

Figure 3 shows $C / T$ versus $T$ for the sample with the second highest RRR of about 185. The Sommerfeld ratio $C / T$, which is enhanced by an order of magnitude over the band structure value of $\simeq 10 \mathrm{~mJ} /\left(\mathrm{molK}^{2}\right)$ $[6,7]$, rises below $T_{c}$, peaks at about $20 \%$ above the normal state value near $0.9 \mathrm{~K}$ and then decreases rapidly (Fig. 3). The heat capacity anomaly is suppressed in applied magnetic field, allowing a view of the underlying normal state. Using the nearly constant normal 


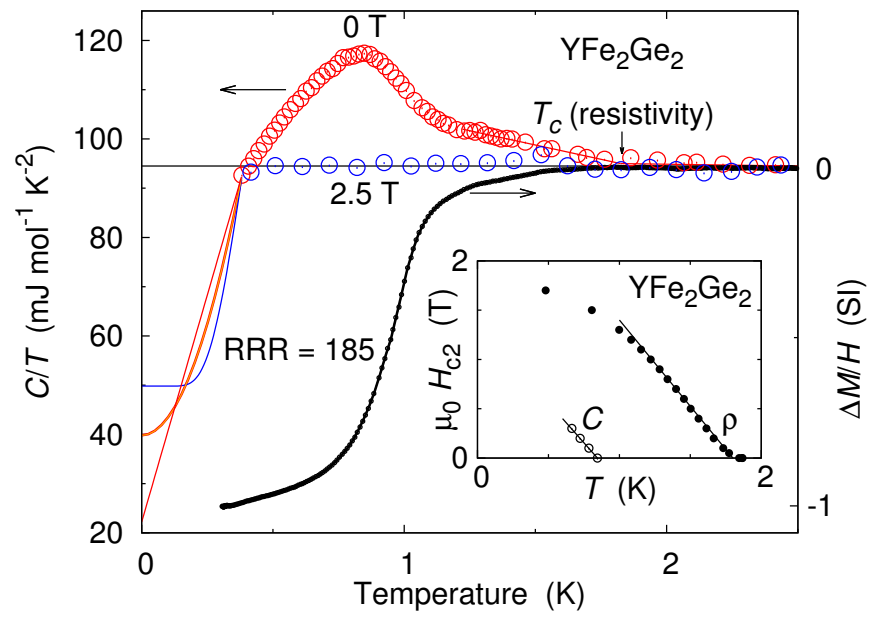

FIG. 3. $C / T$ and magnetisation of $\mathrm{YFe}_{2} \mathrm{Ge}_{2}$ versus temperature show anomalies characteristic of bulk superconductivity. Low temperature extrapolation using linear (red line), quadratic (orange line) and single-gap BCS form (blue line) obeys entropy-matching at $T_{c}$ (see text). The backgroundsubtracted zero-field-cooled low temperature DC magnetisation $\Delta M$ divided by the applied field $\mu_{0} H=0.5 \mathrm{mT}$ of the same sample displays a step with mid-point at $\simeq 0.95 \mathrm{~K}$, which coincides with the steepest descent in $C / T$. The size of the step corresponds to $(95 \pm 5) \%$ diamagnetic screening. (inset) Temperature dependence of the upper critical field determined from the mid-point of resistive transitions (labelled $\rho$ ) and from the peak in $C / T$ (labelled $C$ ).

state Sommerfeld ratio $C / T$ measured in applied field, we can employ an entropy-conserving equal area construction justified by the second-order nature of the superconducting phase transition, in order to obtain estimates of the low temperature residual $C / T$ and thereby of the non-superconducting fraction. [18, 35]. Depending on the assumed low temperature form of the heat capacity, the extrapolated residual $C / T$ reaches $24 \%$, $42 \%$ or $53 \%$ of the normal state value, respectively, for a linear (line-nodes), quadratic (point nodes) or BCSlike (isotropic gap) temperature dependence of $C / T$ [18]. Similar or larger residual $C / T$ fractions were also found in early studies in $\mathrm{Sr}_{2} \mathrm{RuO}_{4}$ [35], in $\mathrm{RbFe}_{2} \mathrm{As}_{2}$ [12] and in $\mathrm{CsFe}_{2} \mathrm{As}_{2}$ [13]. The extrapolated $C / T$ in $\mathrm{YFe}_{2} \mathrm{Ge}_{2}$ places a lower bound of $47 \%$ on the superconducting fraction. However, SQUID magnetometry on the same sample (Fig. 3) reveals a superconducting volume fraction approaching $100 \%$. This discrepancy suggests that the extrapolation of the heat capacity significantly overestimates the actual residual Sommerfeld ratio. If the superconducting gap varies substantially on different sheets of the Fermi surface, as has been proposed for $\mathrm{KFe}_{2} \mathrm{As}_{2}$ [11], this could cause a marked further downturn of the heat capacity below the lowest $T$ measured so far.

More detailed measurements to lower temperature will be necessary to distinguish between gap scenarios, but the present data already rule out alien phases as the origin of the superconducting heat capacity anomaly: pow- der x-ray diffraction limits the alien phase content in the measured sample to about $1 \%$, of which the leading contribution is made by ferromagnetic $\mathrm{Fe}_{0.85} \mathrm{Ge}_{0.15}$ [18]. To obtain an apparent $50 \%$ superconducting fraction from a $1 \%$ alien phase sample fraction would require the alien phase to display a colossal normal state Sommerfeld coefficient of the order of $5 \mathrm{~J} /\left(\mathrm{molK}^{2}\right)$, which would in turn not be consistent with the observed critical field, the expected composition of any alien phase and the magnitude of $T_{c}$.

Further information about the superconducting state can be inferred from its response to applied magnetic field. In our new generation of samples, the initial slope of the resistive upper critical field is determined as $\left|d B_{c 2} / d T\right| \simeq 1.75 \mathrm{~T} / \mathrm{K}$ (inset of Fig. 3). This corresponds to an extrapolated clean-limit weak-coupling orbital-limited critical field $B_{c 2}^{(o)} \simeq 0.73 T_{c}\left|d B_{c 2} / d T\right| \simeq$ $2.3 \mathrm{~T}$ [36], slightly below the value reported in [8] for a sample with a lower $T_{c}$. This discrepancy may be attributed to critical field anisotropy and preferential alignment within our polycrystals. The extrapolated critical field corresponds to a superconducting coherence length $\xi_{0} \simeq 120 \AA$, comparable to the value of $\simeq 180 \AA$ estimated from the field dependence of the heat capacity anomaly, which has an initial slope of $\simeq 1.7 \mathrm{~T} / \mathrm{K}$ (inset of Fig. 3) [18]. Such a short coherence length might be expected to result from the enhanced quasiparticle mass and consequently low Fermi velocity indicated by the high Sommerfeld coefficient. Indeed, the measured Sommerfeld coefficient and the calculated Fermi surface geometry give rise to a short coherence length $\xi_{B C S} \simeq 166 \AA$ [18], in rough agreement with the estimate for $\xi_{0}$ obtained above from the critical field measurement.

The mean free path can likewise be estimated from the low temperature resistivity and the calculated Fermi surface as $\ell \simeq 150 \AA$ for samples with residual resistivity $\rho_{0} \simeq 10 \mu \Omega \mathrm{cm}(\mathrm{RRR} \sim 20)[18]$. The observation that $T_{c}$ correlates with the residual resistance ratio (Fig. 2) and that full transitions are observed in samples for which $\ell>\xi_{0}$ is consistent with unconventional superconductivity [37]. Distinct heat capacity anomalies were only observed in samples with $\rho_{0}<3 \mu \Omega \mathrm{cm}$, corresponding to $\ell>500 \AA$, which exceeds the coherence length by a factor of 3-4. We attribute this primarily to the consequences of sample inhomogeneity, which leads to a distribution of $T_{c}$ within the sample, causing the heat capacity anomaly to be smeared out in all but the best samples. Further complications could arise from multiband superconductivity.

The present thermodynamic evidence establishes superconductivity in $\mathrm{YFe}_{2} \mathrm{Ge}_{2}$ as an intrinsic bulk phenomenon, motivating a more careful look at the likely pairing mechanism. Two theoretical studies $[6,7]$ investigate the electronic structure of $\mathrm{YFe}_{2} \mathrm{Ge}_{2}$, its magnetic properties and the role these could play in determining its superconducting gap structure. Both studies arrive at a Fermi surface structure similar to that shown in Fig. 4. 


\begin{tabular}{|l|l|l|c|c|}
\hline $\mathrm{RFe}_{2} \mathrm{X}_{2}$ & \multicolumn{1}{|c|}{$\begin{array}{c}a \\
(\AA)\end{array}$} & $\begin{array}{c}c \\
(\AA)\end{array}$ & $c / a$ & $\begin{array}{c}\mathrm{X}-\mathrm{X} \\
(\AA)\end{array}$ \\
\hline $\mathrm{YFe}_{2} \mathrm{Ge}_{2}(p=0)$ & $3.964(6)$ & $10.457(4)$ & 2.639 & 2.533 \\
$\mathrm{KFe}_{2} \mathrm{As}_{2}(p=842$ & 13.861 & 3.608 & 4.089 \\
$\mathrm{KFe}_{2} \mathrm{As}_{2}(21 \mathrm{GPa})$ & 3.854 & 9.600 & 2.491 & 2.544 \\
\hline
\end{tabular}

TABLE I. Crystallographic parameters of $\mathrm{YFe}_{2} \mathrm{Ge}_{2}$ at ambient pressure [8], for $\mathrm{KFe}_{2} \mathrm{As}_{2}$ in the ambient pressure uncollapsed tetragonal phase [38] and in the high pressure collapsed tetragonal phase [25]. Comparing the Ge-Ge distance to the As-As distances (both $\mathrm{X}-\mathrm{X}$ ) illustrates the bond formation which accompanies the collapsed tetragonal structure.

The Fermi surface is dominated by a large, disk-shaped hole pocket enclosing the Z-point of the body-centred tetragonal Brillouin zone, as well as a cylindrical electron pocket in the corner of the zone. There are also several smaller hole pockets around the Z-point.

The calculated Fermi surface in $\mathrm{YFe}_{2} \mathrm{Ge}_{2}$ is very similar to that expected for $\mathrm{KFe}_{2} \mathrm{As}_{2}$ in the pressure induced collapsed tetragonal phase (Fig. 4) [39, 40]. This strongly suggests that $\mathrm{YFe}_{2} \mathrm{Ge}_{2}$ is an isoelectronic and isostructural reference compound to collapsed-tetragonal $\mathrm{KFe}_{2} \mathrm{As}_{2}$. The lattice collapse in 1-2-2 arsenides is linked to the formation of As-As bonds [41] and therefore is expected to have profound consequences for the electronic structure, changing the $\mathrm{Fe}$ oxidation state to that of $\mathrm{YFe}_{2} \mathrm{Ge}_{2}$, which features Ge-Ge bonds already at ambient pressure (Tab. I). A pressure induced lattice collapse is known to occur also in $\mathrm{CaFe}_{2} \mathrm{As}_{2}$ [42], which however has a different electron count, causing its high pressure Fermi surface geometry to differ significantly from those of $\mathrm{KFe}_{2} \mathrm{As}_{2}$ and $\mathrm{YFe}_{2} \mathrm{Ge}_{2}[43,44]$.

In view of the recent surprising discovery of superconductivity at enhanced transition temperatures exceeding $10 \mathrm{~K}$ in $\mathrm{KFe}_{2} \mathrm{As}_{2}$ within the collapsed tetragonal phase $[25,39]$, the scenarios for superconductivity in $\mathrm{YFe}_{2} \mathrm{Ge}_{2}$ put forward by Subedi [6] and Singh [7] assume a wider relevance. Whereas the former argues that the presence of an electron pocket at the zone corner and hole pockets near the zone centre favour antiferromagnetic spin fluctuations and an $s_{ \pm}$order parameter wave function, the latter puts forward a more radical proposal: noting that magnetism with ordering wavevector $(0,0,1 / 2)$ can be induced in $\mathrm{YFe}_{2} \mathrm{Ge}_{2}$ by alloying with isoelectronic $\mathrm{Lu}$ $[15,45]$, ferromagnetic correlations within the plane could induce a triplet superconducting state. The $s_{ \pm}$scenario resembles the proposal which has been advanced for high pressure $\mathrm{KFe}_{2} \mathrm{As}_{2}$ [40].

Our experimental results demonstrate that $\mathrm{YFe}_{2} \mathrm{Ge}_{2}$ undergoes a superconducting instability at $T_{c} \simeq 1.8 \mathrm{~K}$ out of a strongly correlated normal state with a high Sommerfeld ratio $\gamma \sim 100 \mathrm{~mJ} /\left(\mathrm{molK}^{2}\right)$ and a non-Fermi liquid form for the temperature dependence of the resistivity, $\rho(T) \simeq \rho_{0}+A T^{3 / 2}$. Together with the strong sensitivity to disorder of the resistive $T_{c}$ and of the heat capacity anomaly this suggests an unconventional pairing

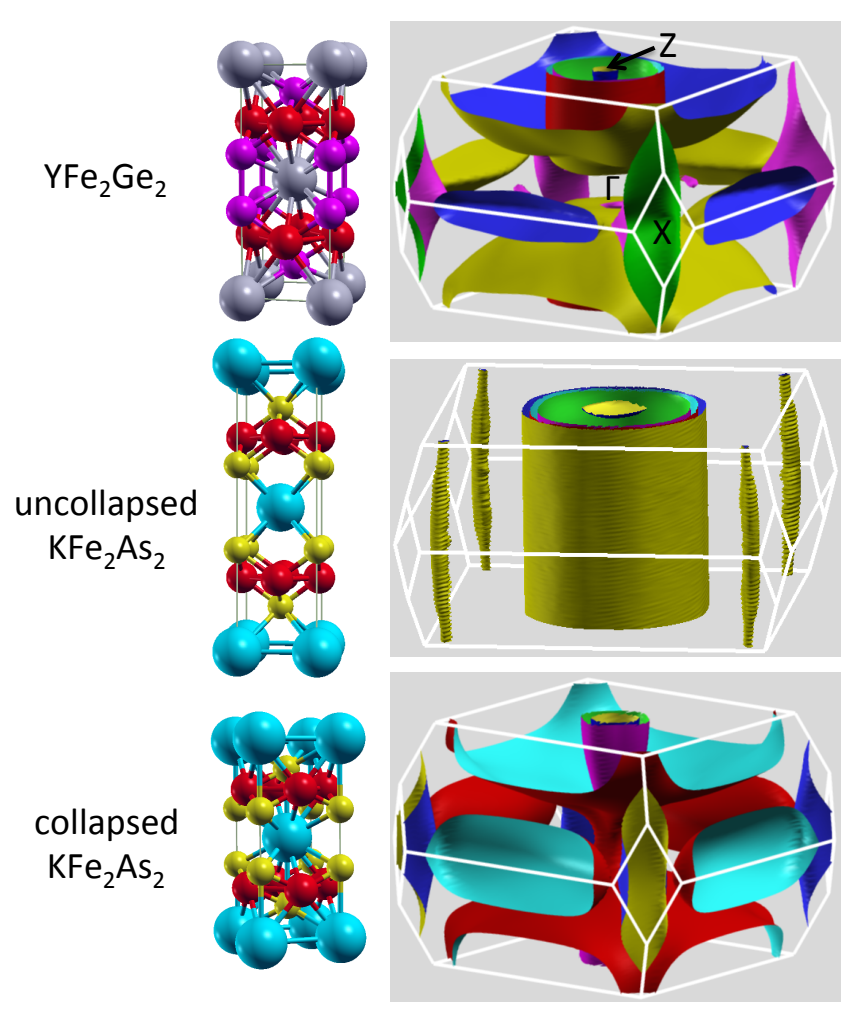

FIG. 4. Fermi surface calculated within DFT for $\mathrm{YFe}_{2} \mathrm{Ge}_{2}$ and for $\mathrm{KFe}_{2} \mathrm{As}_{2}$ in the uncollapsed and collapsed tetragonal structure. The Fermi surfaces of $\mathrm{YFe}_{2} \mathrm{Ge}_{2}$ and uncollapsedtetragonal $\mathrm{KFe}_{2} \mathrm{As}_{2}$ are fundamentally different. Cylindrical hole sheets characterise the Fermi surface structure in uncollapsed-tetragonal $\mathrm{KFe}_{2} \mathrm{As}_{2}$, whereas in $\mathrm{YFe}_{2} \mathrm{Ge}_{2}$, nested $3 \mathrm{D}$ hole pockets on the face of the Brillouin zone $(\mathrm{Z})$ and an electron pocket in the corner of the zone (X) are the main features of the electronic structure near the Fermi energy. Conversely, the Fermi surface of collapsed-tetragonal $\mathrm{KFe}_{2} \mathrm{As}_{2}$ is strikingly similar to that of $\mathrm{YFe}_{2} \mathrm{Ge}_{2}$ apart from the vicinity of the $\Gamma$ point, which is enclosed by an outgrowth of the largest hole pocket in collapsed-tetragonal $\mathrm{KFe}_{2} \mathrm{As}_{2}$, whereas it is avoided by the corresponding hole pocket in $\mathrm{YFe}_{2} \mathrm{Ge}_{2}$.

mechanism. Unconventional superconductivity is rare among transition metal compounds, and $\mathrm{YFe}_{2} \mathrm{Ge}_{2}$ stands out for its more isotropic, 3D Fermi surface, when compared to the cuprates, iron pnictides and chalcogenides, or $\mathrm{Sr}_{2} \mathrm{RuO}_{4}$. The electronic structure of $\mathrm{YFe}_{2} \mathrm{Ge}_{2}$ resembles that of $\mathrm{KFe}_{2} \mathrm{As}_{2}$ in the collapsed tetragonal phase, which can be induced by applied pressure and in which superconductivity with transition temperatures of the order of $10 \mathrm{~K}$ have been reported [25, 39]. This establishes $\mathrm{YFe}_{2} \mathrm{Ge}_{2}$ as a reference compound for investigating the origin of superconductivity in the collapsed tetragonal phase in alkali metal iron arsenides, which is otherwise only accessible at very high applied pressures. 


\section{ACKNOWLEDGMENTS}

We thank, in particular, S. Friedemann, M. Gamza, C. Geibel, P. Niklowitz and G. Lonzarich for helpful discussions, and J. Dann for assistance in aspects of the sample preparation. The work was supported by the EPSRC of the UK and by Trinity College. Supporting data can be found at https://www.repository.cam.ac.uk/handle/1810/253875.

* fmg12@cam.ac.uk

[1] Y. Kamihara, H. Hiramatsu, M. Hirano, R. Kawamura, H. Yanagi, T. Kamiya, and H. Hosono, Journal of the American Chemical Society 128, 10012 (2006).

[2] I. I. Mazin, Nature 464, 183 (2010).

[3] A. P. Mackenzie and Y. Maeno, Reviews Of Modern Physics 75, 657 (2003).

[4] C. W. Chu, L. Z. Deng, and B. Lv, Physica CSuperconductivity 514, 290 (2015).

[5] M. Avila, S. Bud'ko, and P. Canfield, J. Magn. Magn. Mater. 270, 51 (2004).

[6] A. Subedi, Physical Review B - Condensed Matter and Materials Physics 89, 024504 (2014).

[7] D. J. Singh, Physical Review B - Condensed Matter and Materials Physics 89, 024505 (2014).

[8] Y. Zou, Z. Feng, P. W. Logg, J. Chen, G. Lampronti, and F. M. Grosche, Physica Status Solidi - Rapid Research Letters 8, 928 (2014).

[9] H. Kim, S. Ran, E. D. Mun, H. Hodovanets, M. A. Tanatar, R. Prozorov, S. L. Bud'ko, and P. C. Canfield, Philosophical Magazine 95, 804 (2015).

[10] K. Sasmal, B. Lv, B. Lorenz, A. M. Guloy, F. Chen, Y.Y. Xue, and C.-W. Chu, Physical Review Letters 101, 107007 (2008).

[11] F. Hardy, R. Ederl, M. Jackson, D. Aoki, C. Paulsen, T. Wolf, P. Burger, A. Böhmer, P. Schweiss, P. Adelmann, R. A. Fisher, and C. Meingast, Journal of the Physical Society of Japan $\mathbf{8 3}$ (2014).

[12] Z. Zhang, A. F. Wang, X. C. Hong, J. Zhang, B. Y. Pan, J. Pan, Y. Xu, X. G. Luo, X. H. Chen, and S. Y. Li, Physical Review B 91, 024502 (2015).

[13] A. F. Wang, B. Y. Pan, X. G. Luo, F. Chen, Y. J. Yan, J. J. Ying, G. J. Ye, P. Cheng, X. C. Hong, S. Y. Li, and X. H. Chen, Physical Review B 87, 214509 (2013).

[14] F. F. Tafti, A. Ouellet, A. Juneau-Fecteau, S. Faucher, M. Lapointe-Major, N. Doiron-Leyraud, A. F. Wang, X. G. Luo, X. H. Chen, and L. Taillefer, Physical Review B 91, 054511 (2015).

[15] S. Ran, S. L. Bud'ko, and P. C. Canfield, Philosophical Magazine 91, 4388 (2011).

[16] N. Sirica, F. Bondino, S. Nappini, I. Píš, L. Poudel, A. D. Christianson, D. Mandrus, D. J. Singh, and N. Mannella, Physical Review B 91, 121102 (2015).

[17] I. Felner, B. Lv, K. Zhao, and C. W. Chu, Journal of Superconductivity and Novel Magnetism 28, 1207 (2015).

[18] "See Supplemental Material at $[U R L]$, which includes Refs. [19-21], for details of the heat capacity analysis, of powder x-ray diffraction results, and of the calculation of the superconducting coherence length and the mean free path." (2015).
[19] J. S. Kim, E. G. Kim, G. R. Stewart, X. H. Chen, and X. F. Wang, Physical Review B 83, 172502 (2011).

[20] M. Tinkham, Introduction to Superconductivity, Vol. 1 (Dover Publications, 2004).

[21] T. P. Orlando, E. J. McNiff, S. Foner, and M. R. Beasley, Physical Review B 19, 4545 (1979).

[22] H. Enoki, K. Ishida, and T. Nishizawa, Metallurgical Transactions A 18A, 949 (1987).

[23] J. P. Perdew, K. Burke, and M. Ernzerhof, Physical Review Letters 77, 3865 (1996).

[24] P. Blaha, K. Schwarz, G. Madsen, D. Kvasnicka, and J. Luitz, (2001).

[25] J.-J. Ying, L.-Y. Tang, V. V. Struzhkin, H.-K. Mao, A. G. Gavriliuk, A.-F. Wang, X.-H. Chen, and X.-J. Chen, arXiv.org (2015), 1501.00330v1.

[26] C. Pfleiderer, S. Julian, and G. Lonzarich, Nature 414, 427 (2001).

[27] S. Takashima, M. Nohara, H. Ueda, N. Takeshita, C. Terakura, F. Sakai, and H. Takagi, Journal of the Physical Society of Japan 76, 043704 (2007).

[28] R. P. Smith, M. Sutherland, G. G. Lonzarich, S. S. Saxena, N. Kimura, S. Takashima, M. Nohara, and H. Takagi, Nature 455, 1220 (2008).

[29] M. Brando, W. J. Duncan, D. Moroni-Klementowicz, C. Albrecht, D. Gruener, R. Ballou, and F. M. Grosche, Physical Review Letters 101, 026401 (2008).

[30] J. K. Dong, S. Y. Zhou, T. Y. Guan, H. Zhang, Y. F. Dai, X. Qiu, X. F. Wang, Y. He, X. H. Chen, and S. Y. Li, Physical Review Letters 104, 087005 (2010).

[31] X. C. Hong, X. L. Li, B. Y. Pan, L. P. He, A. F. Wang, X. G. Luo, X. H. Chen, and S. Y. Li, Physical Review B 87, 144502 (2013).

[32] F. Hardy, A. E. Böhmer, D. Aoki, P. Burger, T. Wolf, P. Schweiss, R. Heid, P. Adelmann, Y. X. Yao, G. Kotliar, J. Schmalian, and C. Meingast, Physical Review Letters 111, 027002 (2013).

[33] R. Hlubina and T. M. Rice, Physical Review B 51, 9253 (1995).

[34] A. Rosch, Physical Review Letters 82, 4280 (1999).

[35] S. NishiZaki, Y. Maeno, S. Farner, S. I. Ikeda, and T. Fujita, Journal of the Physical Society of Japan 67, 560 (1998); S. NishiZaki, Y. Maeno, and Z. Mao, Journal of Low Temperature Physics 117, 1581 (1999).

[36] E. Helfand and N. R. Werthamer, Physical Review 147, 288 (1966).

[37] A. P. Mackenzie, R. K. W. Haselwimmer, T. A. W, G. G. Lonzarich, Y. Mori, S. NishiZaki, and Y. Maeno, Physical Review Letters 80, 3890 (1998).

[38] S. Rozsa and H.-U. Schuster, Z. Naturforsch. 36b, 1668 (1981).

[39] Y. Nakajima, R. Wang, T. Metz, X. Wang, L. Wang, H. Cynn, S. T. Weir, J. R. Jeffries, and J. Paglione, Physical Review B 91, 060508 (2015).

[40] D. Guterding, S. Backes, H. O. Jeschke, and R. Valentí, Physical Review B 91, 140503 (2015).

[41] R. Hoffmann and C. Zheng, Journal of Physical Chemistry 89, 4175 (1985).

[42] M. S. Torikachvili, S. L. Bud'ko, N. Ni, and P. C. Canfield, Physical Review Letters 101, 057006 (2008).

[43] D. A. Tompsett and G. G. Lonzarich, Physica B 405, 2440 (2010).

[44] A. I. Coldea, C. M. J. Andrew, J. G. Analytis, R. D. McDonald, A. F. Bangura, J. H. Chu, I. R. Fisher, and A. Carrington, Physical Review Letters 103, 026404 (2009). 
[45] T. Fujiwara, N. Aso, H. Yamamoto, M. Hedo, Y. Saiga,
M. Nishi, Y. Uwatoko, and K. Hirota, Journal of the Physical Society of Japan Supplement 76, 60 (2007). 


\section{Superconductivity in the layered iron germanide $\mathrm{YFe}_{2} \mathrm{Ge}_{2}$ Supplemental Material}

\section{ANALYSIS OF HEAT CAPACITY MEASUREMENTS}

In order to extract estimates of the low temperature residual $C / T$, and thereby of the non-superconducting fraction of the sample, we have extrapolated the $C / T$ data subject to a commonly used entropy balancing constraint (see, e.g., [1, 2]): because the superconducting phase transition is second-order, the normal state entropy just above $T_{c}$ has to match the entropy of the superconducting state just below $T_{c}$. To obtain the normal state entropy, we integrate up the heat capacity in applied magnetic fields larger than the upper critical field,

$$
S_{n}\left(T_{c}\right)=\int_{0}^{T_{c}} \frac{C\left(H>H_{c 2}, T\right)}{T} d T,
$$

which can be approximated as $S_{n}\left(T_{c}\right)=\gamma_{0} T_{c}$. Here, $\gamma_{0}$ is the Sommerfeld coefficient in the field-induced normal state, which is taken as constant (Fig. 1).

The superconducting state entropy has a known contribution within the measured temperature range,

$$
S_{1}=\int_{T_{0}}^{T_{c}} \frac{C(H=0, T)}{T} d T,
$$

where $T_{0}$ is the lowest temperature measured. This integral is calculated from the measured data using the trapezoidal method. A further contribution, $S_{0}$, then results from the extrapolation of the heat capacity data to lower temperature. It has to satisfy $S_{0}+S_{1}=S_{n}$. Because above the resistive $T_{c}$ the measured zero-field $C / T$ matches closely the $C / T$ in applied field, the precise choice of the upper limit in the integrals does not affect the calculation of $S_{0}$, as long as the upper limit exceeds the resistive $T_{c}$. Moreover, we constrain the extrapolation to join the measured data point at the lowest measured temperature $T_{0}: C_{0}\left(T_{0}\right)=C\left(H=0, T_{0}\right)$, where $C_{0}(T)$ is the extrapolated heat capacity.

For an initial analysis, we have chosen three forms for the temperature dependence of $C_{0}(T) / T$ :

1. Linear, corresponding to line nodes in the gap function:

$$
C_{0}(T) / T=\alpha_{1} \gamma_{0}+\left(1-\alpha_{1}\right) \beta_{1} T
$$

2. Quadratic, corresponding to point nodes in the gap function:

$$
C_{0}(T) / T=\alpha_{2} \gamma_{0}+\left(1-\alpha_{2}\right) \beta_{2} T^{2}
$$

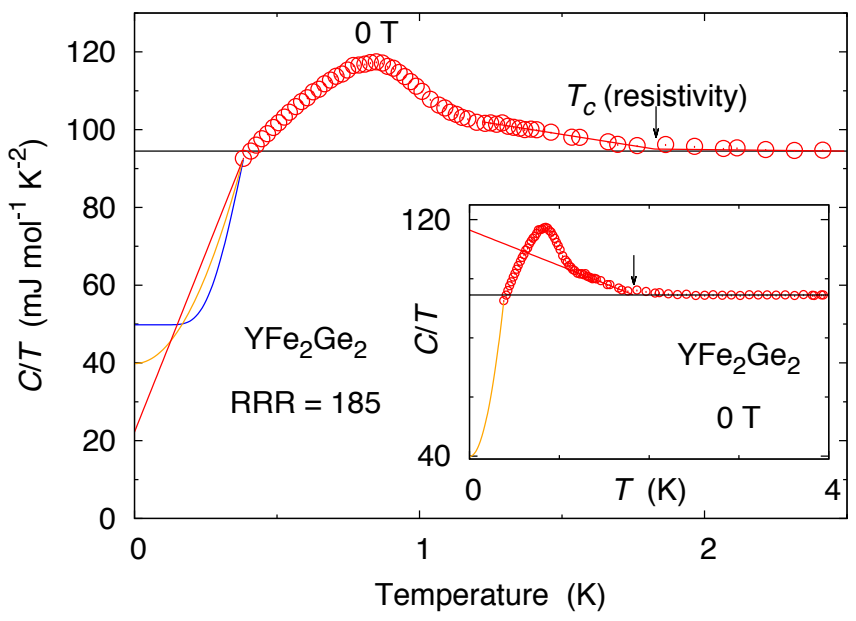

FIG. 1. Illustration of extrapolation schemes for the low temperature heat capacity in $\mathrm{YFe}_{2} \mathrm{Ge}_{2}$. The data is the same as that shown in the manuscript. We compare a linear temperature dependence of $C / T$ (red line), a quadratic $T$-dependence (orange line) and the $T$-dependence expected from BCS theory (blue line). The entropy of normal and superconducting states are matched at $T_{c}$, and the extrapolation function $C_{0}$ matches the measured $C$ at the lowest measured temperature. The normal state $C / T$ is taken from the measurement in high field shown in the manuscript, for which $C / T \simeq 94.5 \mathrm{mJmol}^{-1} \mathrm{~K}^{-2}$. (inset) $C / T$ for the same sample as in the main figure, plotted over a wider $T$-range. The figure illustrates the distinct change in the slope of $C / T$ vs. $T$ near the resistive $T_{c}$.

3. The BCS form for an isotropic gap $\Delta$, which approaches $\Delta=1.76 k_{B} T_{c}^{H C}$ in the low temperature limit, where we retain the freedom to fix a $T_{c}^{H C}$ different from the resistive $T_{c}$ :

$$
C_{0}(T) / T=\alpha_{3} \gamma_{0}+\left(1-\alpha_{3}\right) C_{B C S}\left(T, T_{c}^{H C}\right) / T
$$

Here, $\alpha_{1}, \alpha_{2}$ and $\alpha_{3}$ denote the non-superconducting fractions of the sample in the three cases. The constraints mentioned above, namely (i) matching of normal state entropy and superconducting state entropy at $T_{c}$, and (ii) matching of extrapolation function heat capacity and measured heat capacity at the lowest measured temperature $T_{0}$, make it possible to fix $\alpha$ and $\beta$ in the forms (1) and (2), above, or $\alpha$ and $T_{c}^{H C}$ in the BCS form (3).

A comparison of the three extrapolation schemes is shown in Fig. 1. It demonstrates that independently of the details of the extrapolation scheme, the superconducting fraction is at least of order $50 \%$ of the sample: (i) for the linear extrapolation of $C / T, \alpha_{1}=0.24$; (ii) for the quadratic extrapolation, $\alpha_{2}=0.42$ and (iii) for the BCS form: $\alpha_{3}=0.53$. The BCS extrapolation re- 


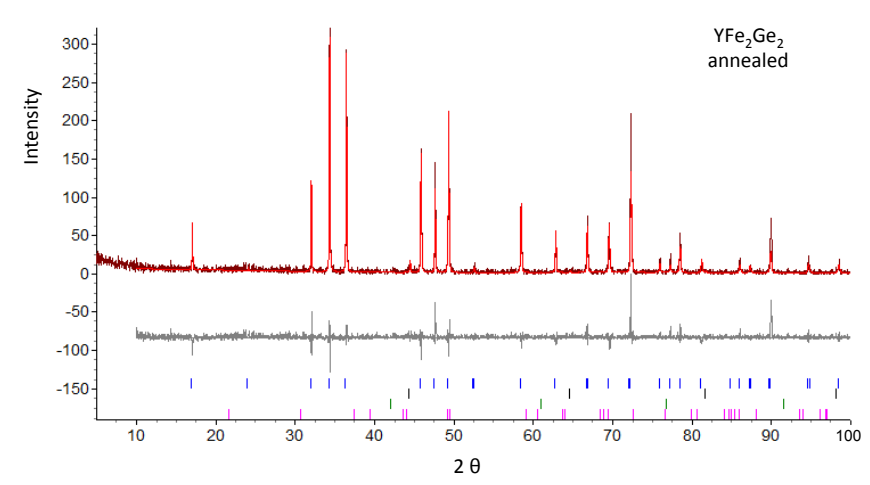

FIG. 2. X-ray pattern obtained on powder from the same annealed ingot from which the heat capacity and transport samples shown in the main part of the manuscript have been extracted.

quired setting a superconducting transition temperature of $T_{c}^{H C}=0.72 \mathrm{~K}$, however, which is well below the peak in the heat capacity plot, casting doubt on its applicability.

The inset of Fig. 1 shows $C / T$ over a wider temperature range, illustrating the change in slope in $C / T$ vs. $T$ near the resistive $T_{c}$. Whereas $C / T$ is nearly constant above $T_{c}$, it rises slowly below $T_{c}$ (orange line on the inset in Fig. 1), before the main heat capacity anomaly is reached. The rise on cooling indicated by the orange line can be attributed to a significant fraction of the sample undergoing the superconducting transition before the main part of the sample, as would be expected from the discrepancy between the resistive $T_{c}$ and the temperature at which the main heat capacity anomaly takes place.

\section{SAMPLE CHARACTERISATION BY POWDER X-RAY DIFFRACTION}

All data were collected in Bragg-Brentano geometry on a D8 Bruker diffractometer equipped with a primary Ge monochromator for $\mathrm{Cu} \mathrm{K}_{\alpha 1}$ and a Sol-X solid state detector to reduce the effects of Fe fluorescence. Figure 2 shows a typical powder pattern, obtained on the ingot from which the samples shown in Figs. 1 and 3 in the main paper were extracted. Collection conditions were: $5-100^{\circ}$ in $2 \theta, 0.03^{\circ}$ step size, 10 seconds/step, divergence slits $0.1 \mathrm{~mm}$, receiving slit $0.2 \mathrm{~mm}$, sample spinning. Rietveld refinements were performed with the software Topas 4.1.

Crystal structures of all phases were retrieved from the inorganic crystal structure database: $\mathrm{YFe}_{2} \mathrm{Ge}_{2}$ (I4/mmm, ICSD reference code: 81745); $\mathrm{Fe}_{0.85} \mathrm{Ge}_{0.15}$ (Im-3m, 103493); Fe (Im-3m, 64795); FeGe 2 (I4/mcm; 42519). A spherical harmonic model was applied to correct for preferred orientation of $\mathrm{YFe}_{2} \mathrm{Ge}_{2}$ within the powder. No structural parameter was refined when resolving the phase content. A shifted Chebyshev function with six parameters was used to fit the background. Peak shapes of all phases were modelled using Pseudo-Voigt functions.

Rietveld refinement of the data obtained from the highest quality samples (Fig. 2) yields: (i) $\mathrm{YFe}_{2} \mathrm{Ge}_{2}$ : $98.7 \%$ and lattice parameters $a=3.963 \AA, c=10.457 \AA$ (blue markers), (ii) $\mathrm{Fe}_{0.85} \mathrm{Ge}_{0.15}$, a bcc iron-germanium alloy with $\mathrm{Im}-3 \mathrm{~m}$ structure: $1.2 \%$ and lattice parameter $2.885 \AA$ (black markers), (iii) iron: $0.1 \%$ and lattice parameter $3.04 \AA$ (green markers), and (iv) traces of $\mathrm{FeGe}_{2}$ (I4mcm) at less than $0.01 \%$ (purple markers). Matching the observed phase content to the nominal composition used in this growth, $\mathrm{YFe}_{2.1} \mathrm{Ge}_{2}$, allows us to estimate the sample composition as $\mathrm{YFe}_{2+x} \mathrm{Ge}_{2}$ with $0<x<0.03$, depending on the precise Fe content in the bcc iron-germanium alloy.

\section{ESTIMATES OF THE SUPERCONDUCTING COHERENCE LENGTH AND OF THE MEAN FREE PATH}

In the standard treatment (e.g. [3]), the orbital-limited resistive critical field in $\mathrm{YFe}_{2} \mathrm{Ge}_{2}$ of $\simeq 2.3 \mathrm{~T}$ corresponds to a superconducting coherence length $\xi_{0}=$ $\left(\Phi_{0} /\left(2 \pi B_{c 2}^{(o)}\right)^{1 / 2} \simeq 120 \AA\right.$, where $\Phi_{0}=h /(2 e)$ is the quantum of flux. By following the peak in $C / T$ with applied field, we can similarly extract an estimate of $\xi_{0}^{C} \simeq 180 \AA$ for the coherence length associated with the bulk transition. We compare this to the BCS coherence length, which we estimate from $\xi_{B C S}=\left(\hbar v_{F}\right) /(\pi \Delta)$ $[3,4]$, where $v_{F}$ is the Fermi velocity and $\Delta$ is the superconducting gap, taken to be $1.76 \mathrm{k}_{\mathrm{B}} T_{c}$. If the electronic structure of $\mathrm{YFe}_{2} \mathrm{Ge}_{2}$ is approximated as an ellipsoidal hole sheet around the $\mathrm{Z}$ point of diameter $2 \AA^{-1}$ and height $0.4 \AA^{-1}$, its enclosed volume is $V_{F} \simeq 1.7 \AA^{-3}$, corresponding to 1.1 carriers per formula unit, and its surface area $S_{F}^{(h)} \simeq 8.2 \AA^{-2}$. The expression for $\xi_{B C S}$ given above can be rewritten in terms of $S_{F}$ as $\xi_{B C S}=$ $V_{0} S_{F} R /\left(1.76 \cdot 12 \pi^{2} \gamma_{0} T_{c}\right)$, where $V_{0}$ is the volume per primitive unit cell, $R$ is the molar gas constant and $\gamma_{0}$ is the normal state Sommerfeld coefficient, giving $\xi_{B C S} \simeq 166 \AA$, in rough agreement with the estimate for $\xi_{0}$ obtained above from the critical field measurement.

The mean free path in our samples can be estimated (e.g. [4]) from the Drude theory result $\ell=$ $6 \pi^{2} h /\left(e^{2} \rho_{0} S_{F}\right)=15,300 \AA\left(\rho_{0} / \mu \Omega \mathrm{cm}\right)^{-1}\left(S_{F} / \AA^{-2}\right)^{-1}$, where $S_{F}$ now includes the total Fermi surface area, which we estimate as $S_{F} \simeq 10 \AA^{-2}$. This gives a mean free path of $\ell \simeq 150 \AA$ for samples with residual resistivity $\rho_{0} \simeq 10 \mu \Omega \mathrm{cm}(\mathrm{RRR} \sim 20)$. 
[1] J. S. Kim, E. G. Kim, G. R. Stewart, X. H. Chen, and X. F. Wang, Physical Review B 83, 172502 (2011).

[2] S. NishiZaki, Y. Maeno, S. Farner, S. I. Ikeda, and T. Fujita, Journal of the Physical Society of Japan 67, 560
(1998).

[3] M. Tinkham, Introduction to Superconductivity, Vol. 1 (Dover Publications, 2004).

[4] T. P. Orlando, E. J. McNiff, S. Foner, and M. R. Beasley, Physical Review B 19, 4545 (1979). 